\title{
EMBEDDED-SIGNAL DESIGN FOR CHANNEL PARAMETER ESTIMATION PART I: LINEAR EMBEDDING
}

\author{
Pierre Moulin \\ University of Illinois \\ Beckman Inst., Coord. Sci. Lab \& ECE Dept. \\ 405 N. Mathews Ave., Urbana, IL 61801 \\ moulin@ifp.uiuc.edu
}

\begin{abstract}
This paper studies the problem of waveform design for identification of communication channel parameters, when the waveform is to be embedded in a host signal. Applications of this study include watermarking and covert communications. We study a subspace-projection embedding method and a penalized nonlinear least-squares parameter estimator. The performance of the estimator is studied in an asymptotic regime. Conditions are given under which the estimator does as well as one that knows the host signal.
\end{abstract}

\section{INTRODUCTION}

In many communication problems, the transmitter and/or receiver's knowledge about the communication channel is limited. In a downlink wireless system, for instance, the channel's gain is time-varying and is unknown to the encoder at the time of transmission. For data communication problems this difficulty may be dealt with using a noncoherent receiver [1]. Alternatively, the receiver may attempt to estimate the unknown channel parameters based on the output and a statistical characterization of the input; this provides a mechanism for channel identification which can be used, e.g., for sending feedback to the transmitter.

To facilitate estimation of channel parameters, one may design input waveforms that are optimized for the estimation task, for instance by maximizing Fisher information. Such strategies have been used to design optimal training sequences [2] and radar waveforms.

The signal design problems that are studied in this paper are somewhat more involved and are motivated by an emerging family of data-embedding applications which includes covert communications, watermarking, inband video captioning, database annotation, etc. [3]. Here the input waveform to be optimized must be a small perturbation embedded within a specified host waveform; the host waveform is often not known to the receiver. A possible approach is one borrowed from traditional point-to-point communications, namely the use of training, or pilot, sequences. This

Work supported by NSF under Grant CCR 00-81268. technique, which is often used for estimating channel gain, delay, or impulse response, may be thought of as waveform embedding using simple time sharing. The use of training sequences has known communication-theoretic disadvantages [4] (ineffective bandwidth utilization and limited adaptivity) as well as security disadvantages in applications such as watermarking. These disadvantages can be remedied by broadening the class of embedding functions. This paper expands our previous work [5] and studies the design and asymptotic performance of linear embedding schemes. A companion paper [6] is devoted to quantization-based embedding schemes.

\section{MATHEMATICAL MODEL}

Consider a linear communication channel with input $x \in$ $\mathbb{R}^{n}$ and output

$$
y=G_{\theta}(x+w), \quad \theta \in \Theta, \quad y \in \mathbb{R}^{n},
$$

where $w \in \mathbb{R}^{n}$ is noise, and $\mathcal{G}=\left\{G_{\theta}, \theta \in \Theta\right\}$ is a given family of $n \times n$ invertible matrices. The parameter set $\Theta$ is a compact subset of $\mathbb{R}^{m}$; the parameter $\theta$ is to be estimated from the channel output. The noise $w$ has zero mean and full-rank covariance matrix $R_{w}$ and is not necessarily Gaussian.

The channel input signal $x$ may not be designed arbitrarily: it must be "close" to a given signal $s \in \mathbb{R}^{n}$, referred to as a host signal. In our model, $s$ is a zero-mean random vector (not necessarily Gaussian) with covariance matrix $R_{s} ; x$ is of the form $x=\Phi(s, p)$, where $p$ is a randomized pilot sequence (known to the receiver), and $\Phi$ satisfies the expected squared-error distortion constraint

$$
\mathbb{E}_{P S}\|X-S\|^{2} \leq n D_{e}
$$

Here $\mathbb{E}_{P S}$ denotes expectation with respect to the distribution of $p$ and $s$, and $D_{e}$ denotes embedding distortion per sample. The estimator of $\theta$ knows $p$, but generally not $s$. Given this model, we want to design the input signal $x$ subject to the distortion constraint (2), so as to accurately estimate $\theta$. 
Examples of (1) may be found in the watermarking and covert communications literature. There the channel (1) is designed by an adversary who attempts to disrupt the transmission of $s$ to the receiver. For many systems, a particularly effective method is a desynchronization attack, in which the attacker implements a channel of the form (1) in which $\theta$ is a delay, a scaling factor, a time-varying delay, an amplitude modulation, a set of filter parameters, etc.

Scaling. Here $G_{\theta}=\theta I_{n}$, where $I_{n}$ is the $n \times n$ identity matrix, and $\Theta=\left[\theta_{\min }, \theta_{\max }\right]$.

Cyclic Delays. A noninteger cyclic delay $\theta \in[0, n]$ is modeled as follows: $G_{\theta}(i, j)=\varphi(\theta+i-j)$ for $1 \leq$ $i, j \leq n$, where $\varphi(t)=\frac{\sin \pi t}{n \sin \pi t / n}$ is the periodic sinc function with period $n$. In other words, $G_{\theta} s(n)$ is a sampled version of the delayed, bandlimited A/D reconstruction of the periodicized signal $s(n)$.

Time-Invariant Filtering. Here $G_{\theta}(i, j)=h_{\theta}(i-j)$, where $h_{\theta}$ is a filter impulse response parameterized by $\theta$. The cyclic-delay model is a special case of this one with $h_{\theta}(i)=\varphi(\theta+i)$.

Note that for the scaling problem, the matrices $G_{\theta}$ are diagonal. For the cyclic-delay problem, one could also work with a frequency representation of the signals, in which case $G_{\theta}$ would be diagonal (with unequal components on the diagonal).

For simplicity of the notation we shall henceforth assume that $\Theta \subset \mathbb{R}$. We also define

$$
\begin{aligned}
H_{\theta} & =G_{\theta}^{-1}, \\
\dot{G}_{\theta} & =\frac{d}{d \theta} G_{\theta}, \\
\dot{H}_{\theta} & =\frac{d}{d \theta} H_{\theta}, \\
Q_{\theta} & =G_{\theta} R_{w} G_{\theta}{ }^{T}, \\
F_{\theta} & =\dot{H}_{\theta} G_{\theta} .
\end{aligned}
$$

The Hilbert-Schmidt norm of a $n \times n$ matrix $A$ is defined as $\|A\|_{H S}=\left((1 / n) \operatorname{Tr}\left[A A^{T}\right]\right)^{1 / 2}$. The Mahalanobis norm of a vector $x$ is defined as $\|x\|_{A}=\left(x^{T} A x\right)^{1 / 2}$, where $A$ is the inverse covariance matrix of $x$. The Gaussian distribution with mean vector $\mu$ and covariance matrix $R$ is denoted by $\mathcal{N}(\mu, R)$.

\section{KNOWN HOST SIGNAL}

In this section we assume that the host $s$ is known to the estimator. Then $x=\Phi(s, p)$ is also known to the estimator and is viewed as deterministic for the analysis below. We select $x=s+p$. Due to (1), we have $\mathbb{E}[Y]=G_{\theta} x$ and $\operatorname{Cov}[Y]=Q_{\theta}$. Consider the penalized nonlinear leastsquares estimator

$$
\begin{aligned}
\hat{\theta}(y, x) & =\operatorname{argmin}_{\theta \in \Theta}\left[\left\|H_{\theta} y-x\right\|_{R_{w}^{-1}}^{2}+2 \ln \left|\operatorname{det} G_{\theta}\right|\right] \\
& =\operatorname{argmin}_{\theta \in \Theta}\left[\left\|y-G_{\theta} x\right\|_{Q_{\theta}^{-1}}^{2}+\ln \operatorname{det} Q_{\theta}\right],(3)
\end{aligned}
$$

which coincides with the maximum-likelihood estimator of $\theta$ when the noise $W$ is $\mathcal{N}\left(0, R_{w}\right)$.

Asymptotic properties (as $n \rightarrow \infty$ ) of the estimator are stated in Theorem 1, under regularity conditions (A1) (A6) below. The dependency of $x, G_{\theta}, F_{\theta}$, etc. on $n$ is implicit.

(A1) $w_{i}, 1 \leq i \leq n$ are i.i.d. $p_{W}$ such that $\mathbb{E} W=\mathbb{E} W^{3}=$ $0, \mathbb{E} W^{2}=\bar{\sigma}_{w}^{2}$, and $\mathbb{E} W^{4}=M_{4} \sigma_{w}^{4}$, where $M_{4}<\infty$.

(A2) $G_{\theta}$ is continuously differentiable with respect to $\theta$.

(A3) $G_{\theta} \neq G_{\theta^{\prime}}$ for any $\theta \neq \theta^{\prime}$.

(A4) $\theta$ is an interior point of $\Theta$.

(A5) There exists $M<\infty$ upper bounding the strong norms of $G_{\theta}, H_{\theta}, \dot{G}_{\theta}$ and $\ddot{G}_{\theta}$, for all $\theta$ and $n$.

(A6) The following limits exist: ${ }^{1}$

$$
\begin{array}{r}
i_{0}(\theta)=\lim _{n \rightarrow \infty} \frac{1}{n}\left\{\operatorname{Tr}\left[F_{\theta}\left(F_{\theta}{ }^{T}+F_{\theta}\right)\right]\right. \\
\left.+\left(M_{4}-3\right) \sum_{i=1}^{n}\left(F_{\theta}\right)_{i i}^{2}\right\} \\
i(\theta, x)=\frac{1}{\sigma_{w}^{2}} \lim _{n \rightarrow \infty} \frac{1}{n}\left\|F_{\theta} x\right\|^{2}+i_{0}(\theta) .
\end{array}
$$

Moreover, $0<i(\theta, x)<\infty$.

Theorem 1. Under assumptions (A1) - (A4), the estimator $\hat{\theta}(y, x)$ is converges in probability to $\theta$. If in addition (A5) and (A6) hold, $\hat{\theta}(y, x)$ is asymptotically normal:

$$
\sqrt{n}(\hat{\theta}-\theta) \sim \mathcal{N}(0, v(\theta)) \quad \text { as } n \rightarrow \infty
$$

(convergence in distribution), where $v(\theta)=\frac{1}{i(\theta, x)}$ is termed the asymptotic variance.

Corollary. If the matrices $G_{\theta}$ are unitary, the penalty term in (3) is independent of $\theta$, and

$$
i(\theta, x)=\frac{1}{\sigma_{w}^{2}} \lim _{n \rightarrow \infty} \frac{1}{n}\left\|F_{\theta} x\right\|^{2} .
$$

Outline of the proof. Referring to (3), denote by $\psi(y, \theta)$ the derivative of the cost function with respect to $\theta$. Define $J_{n}\left(\theta, \theta^{\prime}\right)=\mathbb{E}_{Y \mid \theta} \psi\left(Y, \theta^{\prime}\right)$, which depends only on the second-order statistics of $Y$ (and thus of $W$ ). Assumption (A3) implies that the equation $J_{n}\left(\theta, \theta^{\prime}\right)=0$ admits the single root $\theta^{\prime}=\theta$. Under the regularity conditions (A1) (A4), any sequence of roots of $\psi\left(y, \theta^{\prime}\right)$ converges in probability to its expectation $J_{n}\left(\theta, \theta^{\prime}\right)$ as $n \rightarrow \infty$. The second claim follows from a Taylor series expansion of $\hat{\theta}(y, x)$ around $\theta$ and a Cramer-Wald type analysis [9].

Remark. Under the zero-input condition $x=0$, we have $i(\theta, 0)=i_{0}(\theta)$ which is strictly positive in some cases. However $W$ conveys no information about $\theta$ if $W$ is Gaussian and $\left\{G_{\theta}\right\}$ are unitary.

${ }^{1}$ If $W$ is Gaussian, $M_{4}=3$, and $i(\theta, x)$ is the Fisher information rate. 


\section{PILOT SEQUENCE OPTIMIZATION}

Consider now the following two design problems. In both cases, the pilot sequence $p$ should be independent of $S$ and satisfy the distortion constraint $\|p\|^{2} \leq n D_{e}$.

1. Design $p$ so as to minimize the worst-case (over $\theta$ ) asymptotic variance of the estimator.

2. Design $\theta$ that maximizes the minimal (over $p$ ) asymptotic variance of the estimator.

For a given (large) $n$, we want to solve $\max _{p} \min _{\theta} E_{n}(p, \theta)$ and $\min _{\theta} \max _{p} E_{n}(p, \theta)$, respectively. In many cases the payoff function

$$
\begin{aligned}
E_{n}(p, \theta) & =\frac{1}{\sigma_{w}^{2}} \mathbb{E}_{S} \frac{1}{n}\left\|F_{\theta} x\right\|^{2}+i_{0}(\theta) \\
& =\frac{1}{\sigma_{w}^{2}} \frac{1}{n}\left\{\operatorname{Tr}\left[F_{\theta} R_{s} F_{\theta}^{T}\right]+\left\|F_{\theta} p\right\|^{2}\right\}+i_{0}(\theta)
\end{aligned}
$$

admits a saddlepoint solution $\left(p^{*}, \theta^{*}\right)$ such that

$$
E_{n}\left(p^{*}, \theta^{*}\right)=\max _{p} \min _{\theta} E_{n}(p, \theta)=\min _{\theta} \max _{p} E_{n}(p, \theta) .
$$

Note that

$$
\mathbb{E}_{S} i(\theta, x)=\lim _{n \rightarrow \infty} E_{n}(p, \theta) .
$$

The solution is of the form $p=\sqrt{n D_{e}} \tilde{p}$, where $\tilde{p}$ is any unit-norm vector that achieves $\max _{\tilde{p}} \min _{\theta} \tilde{E}_{n}(\tilde{p}, \theta)$, where

$$
\tilde{E}_{n}(\tilde{p}, \theta):=\frac{1}{\sigma_{w}^{2}} \frac{1}{n} \operatorname{Tr}\left[F_{\theta} R_{s} F_{\theta}^{T}\right]+\frac{D_{e}}{\sigma_{w}^{2}}\left\|F_{\theta} \tilde{p}\right\|^{2}+i_{0}(\theta) .
$$

Solutions can be derived for important special cases:

Scaling operators: it is convenient to use the parameterization $G_{\theta}=e^{\theta} I_{n}, \theta \in \mathbb{R}$, which makes Fisher information for $\theta$ independent of $\theta$. Assume that $\sigma_{s}^{2}=\lim _{n \rightarrow \infty} \frac{1}{n} \operatorname{Tr}\left[R_{s}\right]$ exists and is finite. Then $F_{\theta}=-I_{n}, i_{0}(\theta)=M_{4}-1$, and

$$
\tilde{E}_{n}(\tilde{p}, \theta)=\frac{1}{\sigma_{w}^{2}}\left[\frac{1}{n} \operatorname{Tr}\left[R_{s}\right]+D_{e}\right]+M_{4}-1,
$$

for all $(\tilde{p}, \theta)$. This yields

$$
i(\theta, \tilde{p}):=\mathbb{E}_{S}[i(\theta, x)]=\frac{\sigma_{s}^{2}+D_{e}}{\sigma_{w}^{2}}+M_{4}-1, \quad \forall \theta, \tilde{p} .
$$

Cyclic-delay operators: it is convenient to use a frequencydomain representation for vectors and matrices here. The noise $w$ is Gaussian, and the signals $s, p$ and $w$ are periodic with odd period $n=2 K+1$ and continuous-time Fourier representations of the form

$$
s(t)=\sum_{k=-K}^{K} S_{k} e^{j 2 \pi k t / n}, \quad t \in[0, n] .
$$

Then $G_{\theta}$ is a diagonal matrix with $k$-th diagonal element given by

$$
\left(G_{\theta}\right)_{k k}=e^{-j 2 \pi \theta k / n}, \quad \theta \in[0, n], \quad k \in\{-K, \cdots, K\} .
$$

The formulas above are extended from the real to the complex case. We obtain $i_{0}(\theta) \equiv 0$ (due to the unitariness of $\left.G_{\theta}\right)$ and $\left(F_{\theta}\right)_{k k}=j 2 \pi k / n$ for all $\theta$. If the process $S$ is wide-sense-stationary, its covariance matrix in the frequency domain is diagonal, and we obtain

$$
\tilde{E}_{n}(\tilde{p}, \theta)=\frac{1}{\sigma_{w}^{2}} \frac{1}{n} \sum_{k=-K}^{K}\left(\frac{2 \pi k}{n}\right)^{2}\left(\sigma_{s, k}^{2}+n D_{e} \tilde{p}_{k}^{2}\right) \quad \forall \theta .
$$

The cost function is again independent of $\theta$, and the optimal $\tilde{p}$ is given by $\left\{2^{-1 / 2}, 0, \cdots, 0,2^{-1 / 2}\right\}$. In other words, the optimal $p$ is a sinusoid at the highest allowed frequency. For this problem we obtain

$$
\begin{aligned}
& i(\theta)=\frac{1}{\sigma_{w}^{2}} \lim _{n \rightarrow \infty}\left\{\frac{1}{n} \sum_{k=-K}^{K}\left(\frac{2 \pi k}{n}\right)^{2} \sigma_{s, k}^{2}\right. \\
&\left.+\left(\frac{\pi(n-1)}{n}\right)^{2} D_{e}\right\}, \quad \theta \in \mathbb{R} .
\end{aligned}
$$

For $\tilde{p}$ selected randomly and uniformly on the surface of the unit $n$-dimensional sphere, we obtain

$$
\begin{array}{r}
i(\theta)=\frac{1}{\sigma_{w}^{2}} \lim _{n \rightarrow \infty}\left\{\frac{1}{n} \sum_{k=-K}^{K}\left(\frac{2 \pi k}{n}\right)^{2}\left(\sigma_{s, k}^{2}+D_{e}\right)\right\}, \\
\theta \in \mathbb{R} .
\end{array}
$$

Filtering operators: See [7].

\section{UNKNOWN HOST SIGNAL}

\subsection{Basic Spread-Spectrum System}

The basic spread-spectrum system considered in the watermarking and data hiding literature [3] uses the embedding function $x=s+p$, where $p$ is chosen independently of $s$. Hence estimation accuracy is limited by the presence of the host signal, which is unknown to the estimator and is a substantial source of noise: $\sigma_{s}^{2}>>D_{e}, \sigma_{w}^{2}$ typically. The communication model (1) can be written as $y=G_{\theta}\left(p+w^{\prime}\right)$, where $w^{\prime}=s+w$ is the equivalent noise. The one can apply the results of Sec. 3 to the penalized nonlinear least-squares estimator

$$
\hat{\theta}(y, p)=\operatorname{argmin}_{\theta \in \Theta}\left[\left\|y-G_{\theta} p\right\|_{Q_{\theta}^{-1}}^{2}+\ln \operatorname{det} Q_{\theta}\right],
$$

where $Q_{\theta}=G_{\theta} R_{w^{\prime}} G_{\theta}{ }^{T}$. Under the regularity conditions of Theorem 1, the estimation error is asymptotically Gaussian. The inverse of the asymptotic variance $v(\theta)$ is given by (5) where $x$ is replaced with $p$ and $\sigma_{w}^{2}$ is replaced with $\sigma_{w^{\prime}}^{2}=\sigma_{w}^{2}+\sigma_{s}^{2}$. The asymptotic variance much larger (and unbounded when $\sigma_{s}^{2} \rightarrow \infty$ ) than that in the known hostsignal case. 


\subsection{Subspace Projection Method}

One expects that substantial improvements over the basic spread-spectrum method should be possible using time-sharing or frequency-sharing strategies of the form $x=\tilde{s}+p$, where $\tilde{s}$ is a preprocessed version of $s$, designed to be orthogonal to the synchronization sequence $p$. In particular, choose $\mathcal{P}$ as a low-dimensional subspace of $\mathbb{R}^{n}$, and denote by $\mathcal{P}^{\perp}$ its orthogonal complement, and by $P_{\mathcal{P}}$ the orthogonal projection operator from $\mathbb{R}^{n}$ onto $\mathcal{P}$. Next, choose $p \in \mathcal{P}$ and define $\tilde{s}=s-P_{\mathcal{P}} s \in \mathcal{P}^{\perp}$. If $\mathcal{P}$ is onedimensional, we simply have $\tilde{s}=s-\frac{\langle s, p>}{\|p\|^{2}} p$, a design that has recently been advocated for watermarking applications [8]. ${ }^{2}$ The embedding distortion for our design is $\mathbb{E}\|X-S\|^{2}=\operatorname{Tr}\left[P_{\mathcal{P}} R_{s} P_{\mathcal{P}}\right]+\|p\|^{2}$ and is not allowed to exceed $n D_{e}$. In order to satisfy this constraint, the trace term above should be small enough, and $\mathcal{P}$ should be lowdimensional. ${ }^{3}$

We explore the suitability of such schemes for channel parameter estimation. A desirable property, which we term uniform orthogonality over the family $\mathcal{G}$, would be that the subspaces $\cup_{\theta \in \Theta} G_{\theta} \mathcal{P}$ and $\cup_{\theta \in \Theta} G_{\theta} \mathcal{P}^{\perp}$ remain orthogonal. Then the collection of orthogonal projections $\left\{P_{G_{\theta} \mathcal{P}} y, \theta \in\right.$ $\Theta\}$ of the received data onto the subspaces $G_{\theta} \mathcal{P}$ is a sufficient statistic for the estimation problem, and each $P_{G_{\theta} \mathcal{P}} y$ is free of host-signal interference. One can formally show that a result analogous to Theorem 1 applies: the estimation error is asymptotically Gaussian with the same asymptotic variance as in the known-host case. If the uniformorthogonality property is not satisfied, we have a leakage effect: the received synchronization signal $G_{\theta} p$ is contaminated by the received host signal $G_{\theta} \tilde{s}$.

We now reexamine the two examples considered earlier:

Scaling Operators. Scaling does not affect orthogonality of signals, and thus the uniform-orthogonality property holds regardless of the choice of the subspace $\mathcal{P}$.

Cyclic Delay Operators. Since the Fourier transform diagonalizes $G_{\theta}$ for all $\theta$, it suffices to choose for $\mathcal{P}$ the subspace spanned by selected frequencies, and the uniformorthogonality property holds. As shown in Sec. 3, the most effective frequency is the highest one, and thus the ideal $\mathcal{P}$ is the two-dimensional subspace spanned by the signals $\sin (2 \pi k(n-1) / n)$ and $\cos (2 \pi k(n-1) / n$ for $1 \leq k \leq n$.

Regarding the latter example, note that any choice of $\mathcal{P}$ that is not frequency-selective might be prone to the leakage effect discussed above. But a frequency-selective choice for $\mathcal{P}$ presents substantial disadvantages in applications such as watermarking, where the pilot sequence is easily identified

\footnotetext{
${ }^{2}$ Such schemes are well suited for simple detection problems, in which $G_{\theta}=I_{n}$ and the possible presence of $p$ has to be ascertained from the data. The error exponent for detection is then the same whether or not the host signal $s$ is known to the detector [10].

${ }^{3}$ For instance, if $\operatorname{dim}(\mathcal{P})=k$ and $p$ is uniformly distributed over the surface of the sphere with radius $\sqrt{n D}$, we have $\mathbb{E}\|X-S\|^{2}=$ $k \sigma_{s}^{2}+n D$, whence $D=D_{e}-(k / n) \sigma_{s}^{2}$.
}

by an adversary and could be removed. This problem can be addressed using a nonlinear embedding scheme. Such designs are studied in the companion paper [6]; it is shown that near-ideal host-signal rejection may be achieved again.

\section{REFERENCES}

[1] D. Warrier and U. Madhow, "Spectrally Efficient Noncoherent Communication," IEEE Trans. Info. Thy, Vol. 48, No. 3, pp. 651-668, March 2002.

[2] Y. Jiang, F.-W. Sun and J. S. Baras, "On the Performance Limits of Data-Aided Synchronization," IEEE Trans. Info. Thy, Vol. 49, No. 1, pp. 191-203, Jan. 2003.

[3] I. Cox, M. Miller and J. Bloom, Digital Watermarking, Morgan-Kaufmann, 2002

[4] A. Lapidoth and P. Narayan, "Reliable Communication Under Channel Uncertainty," IEEE Trans. Info. Thy, Vol. 44, No. 6, pp. 2148-2177, Oct. 1998.

[5] P. Moulin and A. Ivanovic, "The Fisher Information Game for Optimal Design of Synchronization Patterns in Blind Watermarking," Proc. IEEE Int. Conf. on Image Processing, Thessaloniki, Greece, Oct. 2001.

[6] P. Moulin, "Embedded-Signal Design for Channel Parameter Estimation. Part II: Quantization-Based Embedding," to appear in Proc. IEEE Statistical Signal Processing Workshop, St Louis, MO, Sep. 2003.

[7] M. A. Thompson, Attack Characterization via Fragile Watermarking, Masters thesis, U. of Illinois at UrbanaChampaign, ECE Dept, Feb. 2003.

[8] H. Malvar and D. Florêncio, "Improved Spread Spectrum: a New Modulation Technique for Robust Watermarking," IEEE Trans. Signal Processing, Vol. 51, No. 4, pp. 898-905, April 2003.

[9] E. L. Lehmann and G. Casella, Theory of Point Estimation, 2nd Ed., Springer-Verlag, 1998.

[10] T. Liu and P. Moulin, "Error Exponents for Watermarking with Squared-Error Distortion Constraints," Proc. ISIT, p. 190, Yokohama, Japan, July 2003. 